\title{
Analytical Model for Sizing the Magnets of Permanent Magnet Synchronous Machines
}

\author{
George Todorov and Bozhidar Stoev \\ Department of Electrical Machines, Faculty of Electrical Engineering, Technical University of Sofia, Sofia 1000, Bulgaria
}

\begin{abstract}
The paper presents a procedure for determining dimensions of magnets that confirm the required characteristics of a PMSM (permanent magnet synchronous motors). The magnet sizes influence on the saliency of the motor and consequently on its parameters and characteristics. An analytical model that accounts the salience, the stator resistance and saturation has been developed. The model uses FEA (finite element analysis) to obtain the stator windings flux linkage, direct-axis and quadrature-axis inductances and phase shift between the quantities. The distribution of the magnetic field produced by the permanent magnets and the magnetic field at operation with rated load has been obtained using this model. An iterative procedure changes the dimensions of the magnets at each step and performs analysis of the model until an operating point with required characteristics is achieved.
\end{abstract}

Key words: Permanent magnets, PMSM, sizing, volume.

\section{Introduction}

The increased efficiency requirements of the international standards for alternating current motors make the designers to look for improved technologies and constructions. PMSM (permanent magnet synchronous motors) give an attractive alternative for a wide range of applications - from line-start constant speed to inverter-fed variable speed drives. Due to their high efficiency, power factor, power and torque density, PMSM are nowadays an important component of high-performance electric drives for electric and hybrid electric vehicles, aircraft, marine, mill, paper etc. applications.

Equations for calculating the size and volume of PM (permanent magnets) have been developed by Balagurov [1] and Gieras [2]. These equations have been originally developed for ferrite and alnico magnets, which possess a non-linear demagnetization characteristic. Nowadays the most commonly used permanent magnets are rare-earth magnets. They characterize with high energy and linear demagnetization characteristic, but a high temperature

Corresponding author: George Todorov, Ph.D., associate professor, research field: electrical machines. sensitivity. Their high energy production allows the designer to use as little permanent magnet material as possible, without sacrificing the performance of the designed motor. Sekerák [3] presents the influence of the magnet material on the volume and size of the magnets. Yoshioka and Morimoto [4] analyze and show the influence of the PM arrangement on the parameters of PMSM for automotive applications and Sorgdrager and Grobler [5] show the influence of the magnets size on the air-gap flux density distribution, but none of them emphasize on sizing of the magnets. Mi [6] presents a sizing procedure for rare-earth magnets ( $\mathrm{NdFeB}$ and $\mathrm{SmCo}$ ) based on the equations of Balagurov and Gieras. Although the procedure developed in Ref. [6] describes a full estimation of the magnets volume and size, some assumptions were made, such as neglecting the pole salience, the stator resistance and saturation. These assumptions can affect the accuracy of calculations and proper size estimation in the design process.

This paper proposes a procedure for sizing rare-earth permanent magnets for PMSM with both salient and non-salient rotor configurations. Using FEA (finite element analysis) and the space vector diagram of the 
PMSM, the saturation and the stator resistance are accounted for in the process of determining a proper volume and size.

\section{Sizing Procedure}

Before starting the procedure the permanent magnets arrangement of the rotor configuration have to be defined. The available choices for PM arrangements are: surface mounted magnets, inset surface mounted magnets, interior radial flux magnets and interior circumferential flux magnets-Fig. 1.

The sizing procedure is subdivided to seven main sections and its structure diagram is shown in Fig. 2.

\subsection{Initial Design Parameters}

After the arrangement of the rotor configuration is chosen the initial design parameters have to be defined. The initial design parameters are divided into two groups. The first group includes the required values of the output power, efficiency and power factor, the phase voltage and frequency, number of pole pairs, the characteristics of the magnets, the stator winding arrangement and its parameters.

The second group of initial parameters includes the stator cross-section, i.e., the outer and inner diameter, the air-gap length, the stator slot dimensions and the rotor shaft diameter. The laminations stack length is also included in the second group. Based on the cross-section dimensions, the maximum possible magnet width $w_{\text {m max }}$ is determined. The width of the magnet per pole, calculated in the following sections must not be larger than the one determined here.

\subsection{Initial Electric Values-Electromagnetic Power and Rated Current}

At this stage of the sizing procedure, the initial values of the electromagnetic power and the rated current have to be defined. The rated current is:

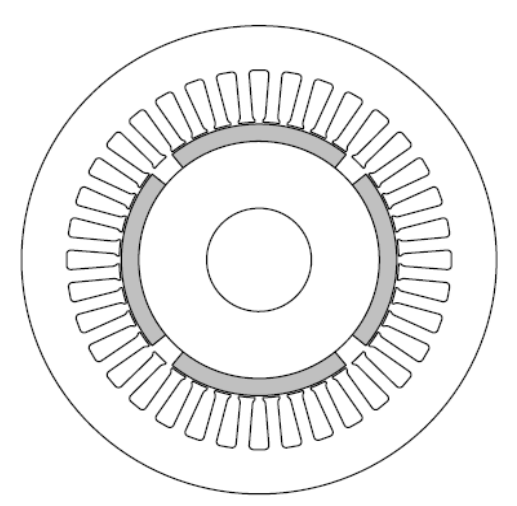

(a)

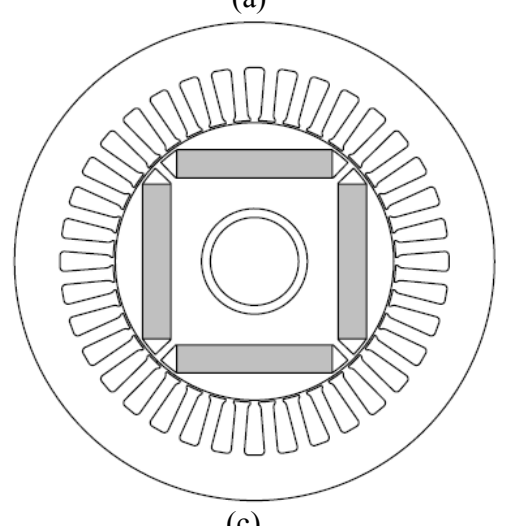

(c)

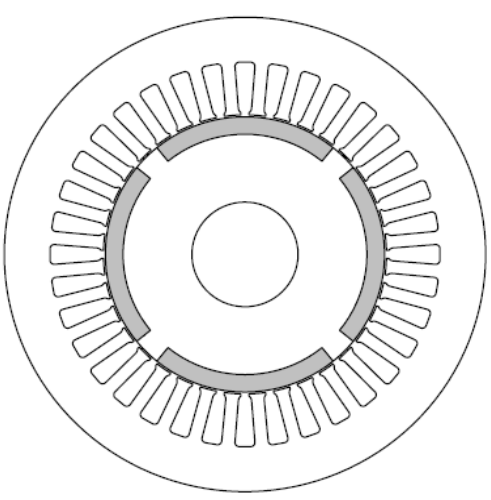

(b)

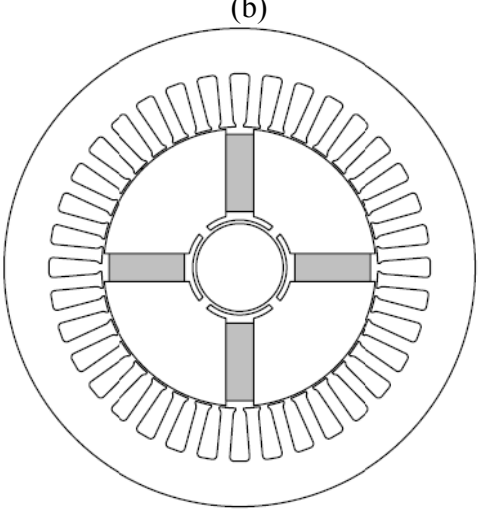

(d)

Fig. 1 Available permanent magnet arrangements: (a) Surface mounted PM; (b) Inset surface mounted PM; (c) Interior radial flux PM; (d) Interior circumferential flux PM. 


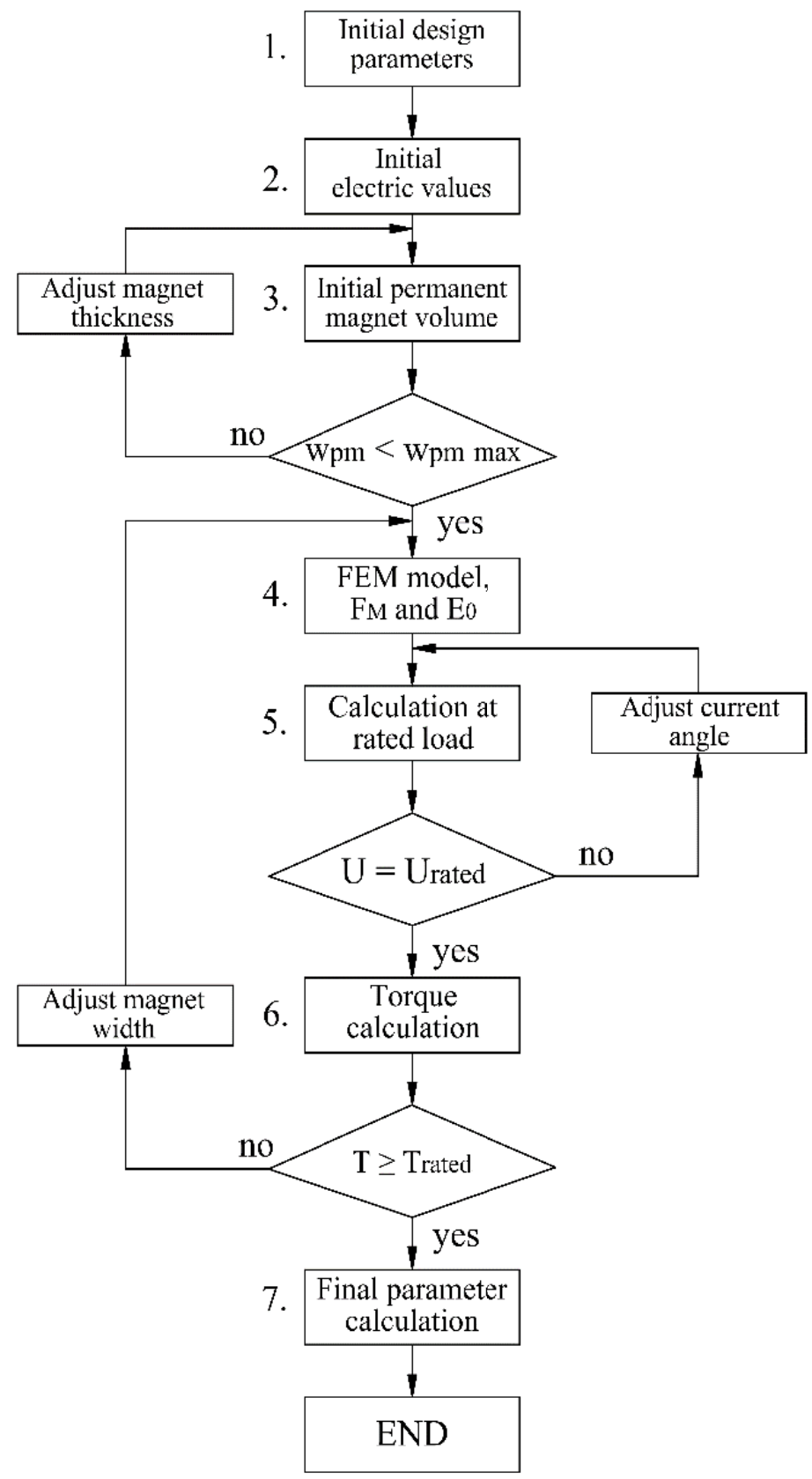

Fig. 2 Procedure structure.

$$
I_{1}=\frac{P_{2}}{m \cdot U_{1} \cdot \eta \cdot \cos \varphi}
$$

where, $m$ is the number of phases, $\eta, \cos \varphi$ are the required values of the efficiency and power factor of the motor.

The electromagnetic power expressed by the input power, efficiency, iron and copper losses is:

$$
P_{\delta}=\frac{P_{2}}{\eta}-\left(p_{e l}+p_{f e}\right)
$$

where, $p_{e l}$ denotes the copper losses and $p_{f e}$-the iron losses.

\subsection{Initial Permanent Magnet Volume}

The relationship between the magnets volume and the electromagnetic power is necessary for defining the initial PM volume.

The electromagnetic power is:

$$
P_{\delta}=m \cdot I_{1} \cdot E_{0} \cdot \cos \beta
$$


where, $E_{0}$ denotes the back e.m.f. induced from the permanent magnets flux and $\beta$ is the current phase angle - the angle between the vectors of the stator current and the back e.m.f (see the space vector diagram of the synchronous motor in Fig. 4).

The back e.m.f. is expressed as:

$$
E_{0}=\sqrt{2} \cdot \pi \cdot W_{1} \cdot k_{W 1} \cdot f_{1} \cdot \phi_{\delta}
$$

In the above equation, $W_{1}$ is the number of turns per phase, $k_{W 1}$ is the winding factor, $f_{1}$ is the frequency of the input voltage and $\phi_{\delta}$ is the air-gap flux per pole.

The rated armature current expressed in terms of the magnetomotive force (m.m.f.) of the direct-axis armature reaction $F_{a d}$ is:

$$
I_{1}=\frac{F_{a d} \cdot p}{0,45 \cdot W_{1} \cdot k_{W 1} \cdot k_{a d} \cdot \sin \beta}
$$

where $k_{a d}$ is the direct-axis armature reaction factor and $p$ is the number of pole pairs.

By substituting Eqs. (4) and (5) into Eq. (3), the following expression for the electromagnetic power is derived:

$$
P_{\delta}=\frac{F_{a d} \cdot p \cdot \sqrt{2} \cdot \pi \cdot W_{1} \cdot k_{W 1} \cdot f_{1} \cdot \phi_{\delta}}{0,45 \cdot W_{1} \cdot k_{W 1} \cdot k_{a d} \cdot \sin \beta}
$$

To calculate the initial volume of the permanent magnets another parameter is needed. It is defined as the magnet usage ratio in Ref. [6], or the coefficient of utilization on the permanent magnets in Ref. [2]:

$$
\xi=\frac{F_{K} \cdot \phi_{M}}{F_{C} \cdot \phi_{r}}
$$

Here $F_{K}$ is the m.m.f. of the magnetic circuit at maximum demagnetizing current $I_{m}=K_{m} \cdot I_{1} ; \phi_{M}$ is the total flux per pole with demagnetizing current zero; $\phi_{r}$ is the total residual flux per pole and $F_{C}$ is the coercive m.m.f. per pole. The definition of the magnet usage ratio is illustrated in Fig. 3.

For surface mounted and interior radial flux magnets, the total flux and the coercive m.m.f per pole are:

$$
\begin{aligned}
& \phi_{r}=B_{r} \cdot w_{m} \cdot l_{m} \\
& F_{C}=2 \cdot H_{C} \cdot h_{m}
\end{aligned}
$$

and for interior circumferential flux magnets:

$$
\phi_{r}=2 . B_{r} \cdot w_{m} \cdot l_{m}
$$

$$
F_{C}=H_{C} \cdot h_{m}
$$

where, $w_{m}$ is the permanent magnet width, $h_{m}$ is the permanent magnet thickness and $l_{m}$ is the length of the permanent magnet along the shaft direction. Usually the magnet length is set to be equal to the laminations stack length. $B_{r}$ and $H_{C}$ are the residual flux density and the coercivity of the magnets.

By expressing the armature reaction from Eq. (7) and substituting in Eq. (6), the following expression for the total volume of the magnets is derived Ref. [6]:

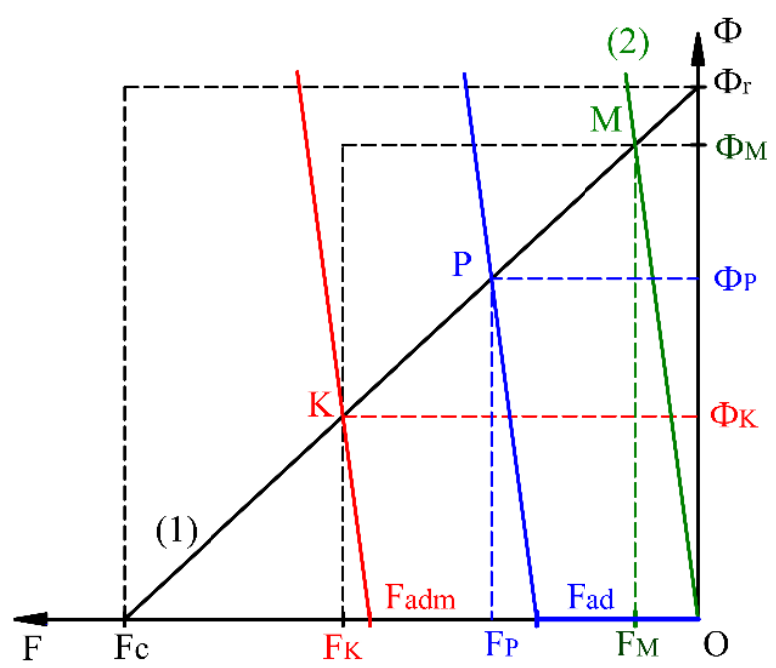

Fig. 3 Illustration of the magnet usage ratio.

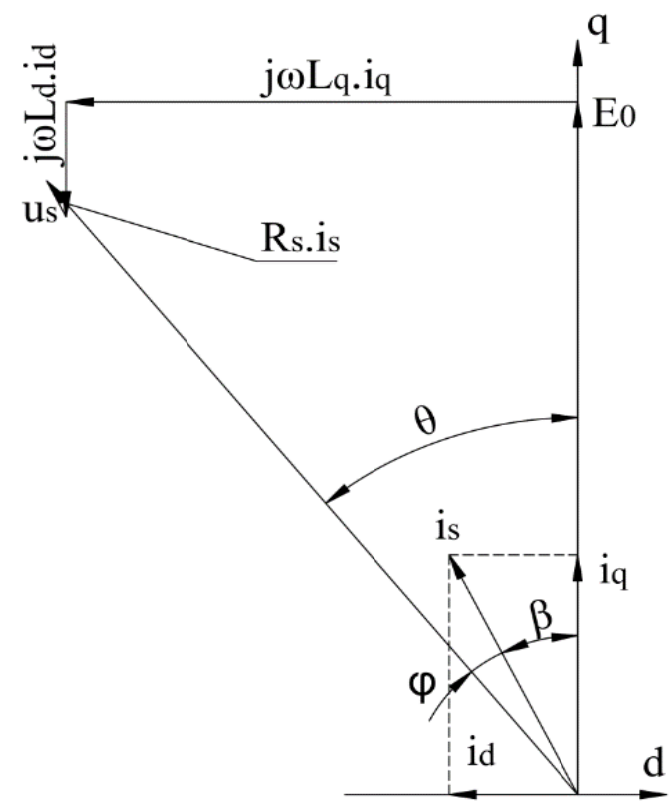

Fig. 4 Space vector diagram of the PMSM. 


$$
V_{m}=\frac{P_{\delta} \cdot 0,45 \cdot k_{a d} \cdot \sin \beta \cdot K_{m} \cdot \sigma_{0}}{\sqrt{2} \cdot \pi \cdot f_{1} \cdot \cos \beta \cdot \xi \cdot H_{c} \cdot B_{r}}
$$

where $K_{m}$ is the maximum armature current per unit and $\sigma_{0}$ is the flux leakage factor.

At this step of the procedure the values of the current angle $\beta$, the leakage factor $\sigma_{0}$, the maximum armature current per unit $K_{m}$ and the direct-axis armature reaction factor $k_{a d}$ are unknown. To calculate an initial volume and size of the magnets, a value has to be assigned to each of the above parameters. The initial values for those parameters are chosen as recommended in the literature $[1,2,6]$. The magnet pole coverage $\alpha_{i}$ should also be assigned for calculating the armature reaction factor $k_{a d}$.

After the volume is determined, the sizes of the magnet per pole are calculated. The thickness of the magnet is:

$$
h_{m}=\tau-\alpha_{i} \cdot \tau
$$

where $\tau$ is the pole pitch.

Since the magnet length is chosen to be equal to the stack length and the magnet thickness is determined from Eq. (13), the width of the magnet can be calculated:

$$
w_{m}=\frac{V_{m}}{2 \cdot p \cdot h_{m} \cdot l_{m}}
$$

The width from Eq. (14) should be compared to the maximum possible width, defined by the cross-section geometry of the motor in the initial design parameters section. If the width is larger than the maximum possible one, the magnet thickness must be readjusted.

\subsection{FEM Model- $F_{M}$ and $E_{0}$ with Zero} Demagnetizing Current

The object of this section is to calculate the e.m.f. $E_{0}$, induced by the permanent magnet flux $\phi_{M}$. The flux $\phi_{M}$ corresponds to the operating point with demagnetizing current zero-point $\mathrm{M}$ in Fig. 3. This is the joint point of the demagnetizing line of the PM (line 1) and the working line that characterizes the permeance of the magnetic circuit with demagnetizing current zero (line 2). The operating point $\mathrm{M}$ is determined via FEA of the motor. Once the initial volume and sizes have been determined, a Finite Element Model of the PMSM is built and analyzed. From the results of the FEA the characteristic of the magnetic circuit and m.m.f $F_{M}$ is determined. The e.m.f. $E_{0}$ is then calculated by substituting the magnetic flux $\phi_{M}$ into Eq. (4).

\subsection{Calculation at Rated Load}

After the m.m.f. and magnetic flux with zero armature current are defined, the operating point of the motor at rated current, for the given permanent magnet volume and dimensions, is determined.

The three phase currents are taken for a moment of time where the current in phase $\mathrm{A}$ is at the maximum and are assigned to the corresponding windings (with the assumption of sinusoidal distribution):

$$
\begin{gathered}
i_{A}=\sqrt{2} \cdot I_{1} \cdot \cos \omega t \\
i_{B}=\sqrt{2} \cdot I_{1} \cdot \cos \left(\omega t-\frac{2 \pi}{3}\right) \\
i_{C}=\sqrt{2} \cdot I_{1} \cdot \cos \left(\omega t-\frac{4 \pi}{3}\right)
\end{gathered}
$$

The phase winding current creates an armature reaction, opposing the field of the permanent magnets. The m.m.f. of the direct axis armature reaction per pole:

$$
F_{a d}=\frac{m \cdot \sqrt{2}}{\pi} \cdot \frac{W_{1} \cdot k_{w 1}}{p} \cdot k_{a d} \cdot i_{d}
$$

This armature reaction tends to magnetize the circuit in a negative direction and moves the operating point of the PM down in the demagnetizing line. Again, the operating point of the magnet is found from the intersection between the demagnetizing line (Fig. 3, line 1) and the working line (line 2). In the case of a rated current and a corresponding armature reaction, the working line 2 is shifted from the origin of the coordinate system with the field strength equal to the demagnetizing m.m.f. of the armature reaction. The operating point $\mathrm{P}$ determines the value of the magnetic flux at rated current $\phi_{P}$, which is used to calculate the back e.m.f. from Eq. (4). 
After performing an FEA with the phase currents assigned to the phase windings, the flux linkages of each phase are found:

$$
\begin{aligned}
& \psi_{A}=W_{c} \cdot \frac{l_{m}}{S_{Z}}\left(\int_{S_{Z A+}} A . d S-\int_{S_{Z A-}} A . d S\right) \\
& \psi_{B}=W_{c} \cdot \frac{l_{m}}{S_{Z}}\left(\int_{S_{Z B+}} A . d S-\int_{S_{Z B-}} A . d S\right) \\
& \psi_{C}=W_{c} \cdot \frac{l_{m}}{S_{Z}}\left(\int_{S_{Z C+}} A . d S-\int_{S_{Z C-}} A . d S\right)
\end{aligned}
$$

where, $W_{c}$ is the number of turns per coil, $l_{m}$ is the lamination stack length, $S_{Z}$ is the cross section of the stator slot and $A$ is the value of the magnetic vector potential for the positive and negative sides of the coils respectively.

To determine the parameters of the motor for the rated operating point, a coordinate system fixed to the rotor, i.e., d-q coordinate system and Park transformations are used. The direct-axis and quadrature-axis components of the current space vector are calculated by:

$$
\begin{gathered}
i_{d}=\frac{2}{3} \cdot\left[i_{A} \cdot \cos \left(\theta_{r}\right)+i_{B} \cdot \cos \left(\theta_{r}-\frac{2 \pi}{3}\right)\right. \\
\left.+i_{C} \cdot \cos \left(\theta_{r}-\frac{4 \pi}{3}\right)\right] \\
i_{q}=-\frac{2}{3} \cdot\left[i_{A} \cdot \sin \left(\theta_{r}\right)+i_{B} \cdot \sin \left(\theta_{r}-\frac{2 \pi}{3}\right)\right. \\
\left.+i_{C} \cdot \sin \left(\theta_{r}-\frac{4 \pi}{3}\right)\right]
\end{gathered}
$$

The $\mathrm{d}$-axis and q-axis components of the stator flux linkage are then determined by:

$$
\begin{gathered}
\psi_{d}=\frac{2}{3} \cdot\left[\psi_{A} \cdot \cos \left(\theta_{r}\right)+\psi_{B} \cdot \cos \left(\theta_{r}-\frac{2 \pi}{3}\right)+\right. \\
\left.+\psi_{C} \cdot \cos \left(\theta_{r}-\frac{4 \pi}{3}\right)\right] \\
\psi_{q}=-\frac{2}{3} \cdot\left[\psi_{A} \cdot \sin \left(\theta_{r}\right)+\psi_{B} \cdot \sin \left(\theta_{r}-\frac{2 \pi}{3}\right)\right. \\
\left.+\psi_{C} \cdot \sin \left(\theta_{r}-\frac{4 \pi}{3}\right)\right]
\end{gathered}
$$

where $\theta_{r}$ is the rotor position angle (the angle between the $\mathrm{d}$-axis and the magnetic axis of phase $\mathrm{A}$ ). The $\mathrm{d}$-axis and q-axis inductances of the motor are:

$$
\begin{gathered}
L_{d}=\frac{\left(\psi_{d}-\psi_{M}\right)}{i_{d}} \\
L_{q}=\frac{\psi_{q}}{i_{q}}
\end{gathered}
$$

where $\psi_{M}$ is the stator winding flux linkage obtained for the operating point $\mathrm{M}$.

The d-axis and q-axis synchronous reactances are:

$$
\begin{gathered}
x_{d}=\omega_{1} \cdot L_{d} \\
x_{q}=\omega_{1} \cdot L_{q}
\end{gathered}
$$

where $\omega_{1}$ is the angular frequency.

The determination of the operating point of the motor at rated load is done by an iterative procedure for defining the values of $i_{d}, i_{q}, L_{d}, L_{q}$, the current phase angle $\beta$ and the load angle $\theta$. The procedure stops when such values are determined, that balance the voltage equation

$$
u_{s}=E_{0}+j \omega L_{d} \cdot i_{d}+j \omega L_{q} \cdot i_{q}+i_{s} \cdot R_{s}
$$

that is graphically depicted with the space vector diagram in Fig. 4.

If the current values of the quantities do not balance Eq. (30) the current phase angle must be readjusted. The correct phase angle is determined by an iterative loop. For each new value of the angle, the phase currents, magnet operating point, flux linkages and inductances are recalculated using FEA. The loop stops when the voltage drops balance out the input voltage.

\subsection{Torque Calculation}

This section checks whether the obtained magnet volume and sizes will provide enough energy to produce the required output torque and power. The electromagnetic torque is computed using the values of $i_{d}, i_{q}, L_{d}, L_{q}$ obtained for the rated point:

$$
T=\frac{3}{2} \cdot p \cdot\left[\psi_{P M} \cdot i_{q}+\left(L_{d}-L_{q}\right) \cdot i_{d} \cdot i_{q}\right]
$$

and the electromagnetic power is:

$$
P_{\delta}^{\prime}=\frac{T \cdot 2 \cdot \pi \cdot n_{1}}{60}
$$

where $n_{1}$ is the synchronous speed of the motor in turn per minute.

If the power calculated from Eq. (32) is lower than 
the one calculated from Eq. (2) in section 2, the width of the magnets must be adjusted. Based on the difference between the previously defined power and the one computed here a new width of the magnets can be found as:

$$
w_{m(1)}=w_{m}-\left(w_{m} \cdot \frac{P_{\delta}^{\prime}-P_{\delta}}{P_{\delta}}\right)
$$

where $w_{m}$ is the magnet width calculated from Eq. (14), $P_{\delta}$ is the required electromagnetic power from Eq. (2) and $P_{\delta}^{\prime}$ is the power calculated from Eq. (32).

When a new magnet width is found, the model has to be rebuild and the calculations have to be repeated starting from section 4 . The iterative procedure for new magnet width stops when such dimensions are found, that satisfy the required electromagnetic power and provide the proper work of the motor.

\subsection{Final Parameter Calculation}

Having the dimensions of the PM, the values of all quantities, the current phase angle $\beta$ and the load angle $\theta$ at rated operating point, the efficiency and power factor are calculated. At the end of the sizing procedure the final values for the initially assigned leakage factor $\sigma_{0}$, maximum per unit armature current $K_{m}$ and the magnet usage ratio $\xi$ are calculated.

The leakage factor $\sigma_{0}$ is calculated as:

$$
\sigma_{0}=\frac{\phi_{M}}{\phi_{P}}
$$

where $\phi_{P}$ is the air-gap flux at the rated operating point of the motor.

The maximum armature current occurs, when the voltage is out of phase with the back e.m.f., i.e.:

$$
I_{\max }=\frac{E_{0}+U}{x_{d}}
$$

The per unit maximum current is defined as:

$$
K_{m}=\frac{I_{\max }}{I_{1}}
$$

The final value of the magnet usage ratio $\xi$ is calculated from Eq. (7) for the operating point $\mathrm{K}$ with armature reaction m.m.f. at maximum demagnetizing current:

$$
F_{a d m}=\frac{m \cdot \sqrt{2}}{\pi} \cdot \frac{W_{1} \cdot k_{w 1}}{p} \cdot k_{a d} \cdot i_{d} \cdot K_{m}
$$

\section{Design Example}

To illustrate the sizing procedure described above, a $1.5 \mathrm{~kW}, 1,500 \mathrm{rpm}$, 4-pole, three phase interior PM synchronous motor is chosen and its magnets are sized according to the required characteristics.

Efficiency of $92 \%$ and power factor of 0.96 are required at rated power and speed. Magnets used for excitation are circumferentially magnetized $\mathrm{NdFeB}$ permanent magnets, with a residual flux density $\mathrm{B}_{\mathrm{r}}=1.05 \mathrm{~T}$ and coercivity $\mathrm{H}_{\mathrm{C}}=0.764 \mathrm{kA} / \mathrm{m}$.

The main initial design parameters are: stator outer diameter $D_{a 1}=136 \mathrm{~mm}$, stator inner diameter $D_{1}=$ $80 \mathrm{~mm}$, air-gap length $\delta=0.55 \mathrm{~mm}$, lamination stack length $l_{f e}=80 \mathrm{~mm}$ and turns per phase $W_{1}=336$.

By using the proposed sizing procedure, the following magnet volume and sizes are determined:

PM volume $V_{m}=42,23 \cdot 10^{-6} \mathrm{~m}^{3}$;

magnet width $w_{m}=21 \mathrm{~mm}$;

magnet thickness $h_{m}=6 \mathrm{~mm}$;

magnet length $l_{m}=80 \mathrm{~mm}$;

Fig. 6 shows the flux distribution from the final FEA solution.

The values of the initial parameters, used to define an initial magnet volume, and the final values calculated at the end of the sizing procedure are shown in Table 1.

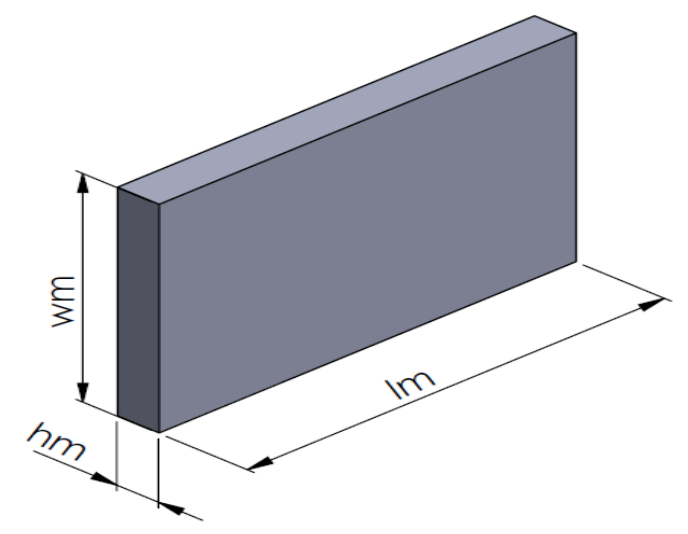

Fig. 5 Permanent magnet dimensions. 


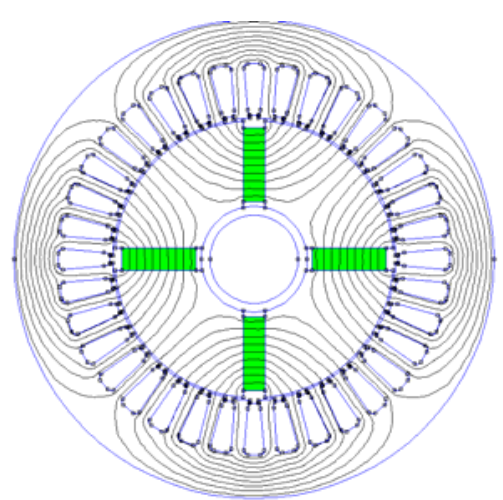

(a)

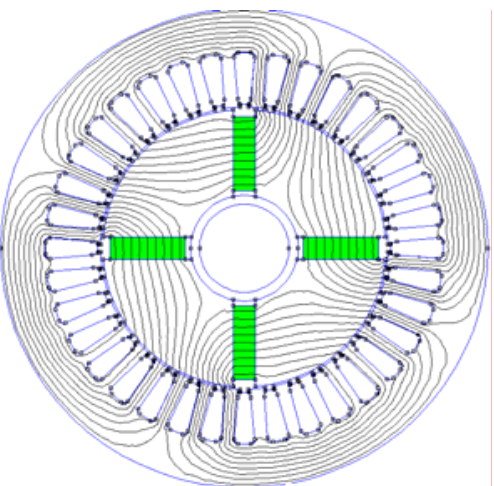

(b)

Fig. 6 Flux distribution: (a) with armature current zero; (b) at rated armature current.

Table 1 Parameter values for the design example.

\begin{tabular}{|c|c|c|c|c|c|}
\hline$\beta$ & $\sigma_{0}$ & $K_{m}$ & $\xi$ & \multicolumn{3}{l|}{$\cos \varphi$} & $\eta$ \\
\hline Initial values & 1.2 & 4 & 0.6 & 0.96 & $92 \%$ \\
\hline $35^{0}$ & & & & \\
\hline Final values & 1.13 & 3.2 & 0.534 & 0.957 & $91.8 \%$ \\
\hline $23^{0}$ &
\end{tabular}

\section{Conclusions}

This paper presents an analytical procedure for determining the volume and size of the permanent magnets of a PMSM. As the PMSM characteristics are strongly influenced by the characteristics and size of the magnets, it is important to know the application of the motor. The core idea is to determine such volume and size that will satisfy the initial requirements, provide the proper work of the motor in the whole range of operating modes and use minimum permanent magnet material.

The model accounts the saliency of the motor, the stator resistance and uses FEA which has made it possible to automatically include the saturation of all parts of the magnetic circuit. The procedure requires number of iterations to achieve the required solution.

Performing the design using model of the motor that apply FEA combined with the vector diagrams and Park transformations allows the sizing of the permanent magnets with good accuracy, verified by the obtained results.

\section{References}

[1] Balagurov, V. A., Galtieev, F. F., and Larionov, A. N. 1964. Permanent Magnet Electrical Machines (in Russian, and translation in English). Moscow, Russia: Energia.

[2] Gieras, J. F., and Wing, M. 2002. Permanent Magnet Motor Technology: Design and Applications. 2nd ed. New York: Marcel Dekker.

[3] Sekerák, P., and Hrabovcová, V. 2012. "Synchronous Motors with Different PM Materials.” ELEKTRO 2012, 241-6.

[4] Yoshioka, S., and Morimoto, S. 2014. "Influence of Magnet Arrangement on the Performance of IPMSMs for Automotive Applications." Energy Conversion Congress and Exposition (ECCE) 2014 IEEE, 4507-12.

[5] Sorgdrager, A., and Grobler, A. 2013. "Influence of Magnet Size and Rotor Topology on the Air-Gap Flux Density of a Radial Flux PMSM." IEEE International Conference on Industrial Technology (ICIT) 2013, 337-43.

[6] Mi, C. C. 2006. "Analytical Design of Permanent Magnet Traction-Drive Motors." IEEE Transactions on Magnetics, 42 (7): 1861-6. 